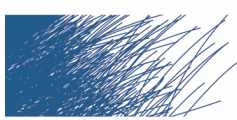

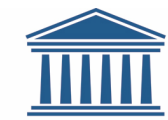 \\ UNIVERSITEIT \\ GENT
}

biblio.ugent.be

The UGent Institutional Repository is the electronic archiving and dissemination platform for all UGent research publications. Ghent University has implemented a mandate stipulating that all academic publications of UGent researchers should be deposited and archived in this repository. Except for items where current copyright restrictions apply, these papers are available in Open Access.

This item is the archived peer-reviewed author-version of:

Title: On the support of tempered distributions

Authors: Jasson Vindas and Ricardo Estrada

In: Proceedings of the Edinburgh Mathematical Society (Series 2), 53 (1), 255-270, 2010

Optional: $\underline{\text { doi:10.1017/S0013091508000102 }}$

To refer to or to cite this work, please use the citation to the published version:

J. Vindas, R. Estrada (2010). On the support of tempered distributions. Proc. Edinb. Math. Soc.(2) 53 (1) 255-270. doi:10.1017/S0013091508000102 


\title{
ON THE SUPPORT OF TEMPERED DISTRIBUTIONS
}

\author{
JASSON VINDAS AND RICARDO ESTRADA
}

\begin{abstract}
In this article we show that if the summability means in the Fourier inversion formula for a tempered distribution $f \in$ $\mathcal{S}^{\prime}\left(\mathbb{R}^{n}\right)$ converge to zero pointwise in an open set $\Omega$, and if those means are locally bounded in $L^{1}(\Omega)$, then $\Omega \subset \mathbb{R}^{n} \backslash \operatorname{supp} f$. We prove this for several summability procedures, in particular for Abel summability, Cesàro summability, and Gauss-Weierstrass summability.
\end{abstract}

\section{INTRODUCTION}

In a recent study, González Vieli and Graham [7] characterized the support of certain tempered distributions in several variables in terms of the uniform convergence over compacts of the symmetric Cesàro means of its Fourier inversion formula. Indeed, they proved that for a large class of tempered distributions $f \in \mathcal{S}^{\prime}\left(\mathbb{R}^{n}\right)$, if for some $k \in \mathbb{N}$

$$
\lim _{a \rightarrow \infty} \int_{|\mathbf{u}| \leq a} \widehat{f}(\mathbf{u}) e^{-i \mathbf{u} \bullet \mathbf{x}} \mathrm{d} \mathbf{u}=0 \quad(\mathrm{C}, k),
$$

uniformly on compacts of an open set $\Omega \subset \mathbb{R}^{n}$, then $\Omega \subset \mathbb{R}^{n} \backslash \operatorname{supp} f$. See also $[6,8,9]$. Results on this subject have a rich tradition that goes back to the work of Kahane and Salem [10] and that of Walter [18]. Here we use the constants in the Fourier transform such that $\widehat{f}(\mathbf{u})=\int_{\mathbb{R}^{n}} f(\mathbf{x}) e^{i \mathbf{u} \mathbf{0} \times} \mathrm{d} \mathbf{x}$ if the integral exists. Hence, the inversion formula becomes $f(\mathbf{x})=(2 \pi)^{-n} \int_{\mathbb{R}^{n}} \widehat{f}(\mathbf{u}) e^{-i \mathbf{u} \bullet \mathbf{x}} \mathrm{d} \mathbf{u}$ when the integral makes sense. If instead of uniform convergence one has only pointwise convergence, then it is easy to see that maybe $\Omega \cap \operatorname{supp} f \neq \varnothing$.

The aims of this note are the following:

(1) To obtain the characterization of the support of any tempered distribution.

2000 Mathematics Subject Classification. 42B10, 46F10, 42B08, 40C99.

Key words and phrases. Support of tempered distributions; Fourier transforms; Cesro summability; Abel summability; spherical means. 
(2) To prove the result under weaker conditions than uniform convergence of the means, in particular, when the means are locally $L^{1}$ bounded.

(3) To obtain the corresponding result for other summability methods such as Abel summability and Gauss-Weierstrass summability.

It should be pointed out that in the one-variable case one can completely characterize the support of a tempered distribution in term of the pointwise Cesàro behavior if one uses slightly asymmetric means. It was proved in [2] that a periodic distribution of period $2 \pi, f \in \mathcal{S}^{\prime}(\mathbb{R})$, with Fourier series $\sum_{n=-\infty}^{\infty} a_{n} e^{i n x}$, has the distributional point value $f\left(x_{0}\right)=\gamma$ in the Eojasiewicz sense [11] if and only if there exists $k$ such that $\forall a>0$,

$$
\lim _{y \rightarrow \infty} \sum_{-a y \leq n \leq y} a_{n} e^{i n x_{0}}=\gamma \quad(\mathrm{C}, k) .
$$

This result was recently generalized to arbitrary tempered distributions $[15,16]:$ If $f \in \mathcal{S}^{\prime}(\mathbb{R})$ then

$$
f\left(x_{0}\right)=\gamma \quad \text { distributionally, }
$$

if and only if

$$
\text { e.v. }\left\langle\widehat{f}(u), e^{-i u x_{0}}\right\rangle=2 \pi \gamma \quad(\mathrm{C}, k) \text {. }
$$

The slightly asymmetric evaluation e.v. $\langle g(x), \rho(x)\rangle(\mathrm{C}, k)$ of a distribution $g \in \mathcal{D}^{\prime}(\mathbb{R})$ on a test function $\rho \in \mathcal{E}(\mathbb{R})$ exists and equals $L$ if for each primitive $G$ of $\rho g, G^{\prime}=\rho g$, we have that $\forall a>0$,

$$
\lim _{y \rightarrow \infty}(G(y)-G(-a y))=L \quad(\mathrm{C}, k) .
$$

Therefore, since the Eojasiewicz point values determine a distribution completely if they exist at all points [11], we obtain the following characterization of the support of a distribution.

Theorem 1. Let $f \in \mathcal{S}^{\prime}(\mathbb{R})$. Let $\Omega$ be an open set of $\mathbb{R}$. If there exists $k$ such that

$$
\text { e.v. }\left\langle\widehat{f}(u), e^{-i u x}\right\rangle=0 \quad(C, k), \quad \forall x \in \Omega,
$$

then $\Omega \subset \mathbb{R} \backslash \operatorname{supp} f$.

The principal value evaluation p.v. $\langle g(x), \rho(x)\rangle(\mathrm{C}, k)$ of a distribution $g \in \mathcal{D}^{\prime}(\mathbb{R})$ on a test function $\rho \in \mathcal{E}(\mathbb{R})$ exists and equals $L$ if for each primitive $G$ of $\rho g, G^{\prime}=\rho g$, we have that

$$
\lim _{y \rightarrow \infty}(G(y)-G(-y))=L \quad(\mathrm{C}, k) .
$$


Naturally the Theorem 1 is not true for principal value evaluations, as the example $f(x)=\delta^{\prime}(x)$ shows, since here the means converge to zero in the p.v. sense for all $x \in \mathbb{R}$.

The plan of the article is as follows. In Section 2 we recall the definitions of Łojasiewicz point values and of Cesàro behavior. Section 3 gives the definition of the distributional $\phi$-transform in several variables and the proof of the distributional convergence. The basic summability procedures for the Fourier inversion formula, and their relation with the distributional $\phi$-transform are presented in Section 4; we observe that summability results for the Fourier transform and its inverse can be considered as particular cases of results for the distributional $\phi$-transform. In Section 5 we show the uniform convergence on compacts of the distributional $\phi$-transform of a function continuous in an open set and its converse, and consequently for summability in the Fourier inversion formula. Finally in Section 6 we characterize the complement of the support of a distribution in the case when the means are locally $L^{1}$ bounded.

\section{Preliminaries}

The spaces of test functions $\mathcal{D}, \mathcal{E}$, and $\mathcal{S}$ and the corresponding spaces of distributions are well-known [5]. We shall also need the distribution space $\mathcal{K}^{\prime}$, the dual of $\mathcal{K}$; the space $\mathcal{K}^{\prime}$ plays a fundamental role in the theory of summability of distributional evaluations [5].

We shall use the notion of the distributional point value of generalized functions introduced by Łojasiewicz, in one [11] and several variables [12]. Let $f \in \mathcal{D}^{\prime}\left(\mathbb{R}^{n}\right)$, and let $\mathbf{x}_{0} \in \mathbb{R}^{n}$. We say that $f$ has the distributional point value $\gamma$ at $\mathbf{x}=\mathbf{x}_{0}$, and write

$$
f\left(\mathbf{x}_{0}\right)=\gamma, \text { distributionally },
$$

if $\lim _{\varepsilon \rightarrow 0} f\left(\mathbf{x}_{0}+\varepsilon \mathbf{x}\right)=\gamma$ in the space $\mathcal{D}^{\prime}\left(\mathbb{R}^{n}\right)$, that is, if

$$
\lim _{\varepsilon \rightarrow 0}\left\langle f\left(\mathbf{x}_{0}+\varepsilon \mathbf{x}\right), \phi(\mathbf{x})\right\rangle=\gamma \int_{\mathbb{R}^{n}} \phi(\mathbf{x}) \mathrm{d} \mathbf{x},
$$

for all test functions $\phi \in \mathcal{D}\left(\mathbb{R}^{n}\right)$. It can be shown that $f\left(\mathbf{x}_{0}\right)=\gamma$, distributionally, if and only if there exists a multi-index $\mathbf{k}_{0} \in \mathbb{N}^{n}$ such that for all multi-indices $\mathbf{k} \geq \mathbf{k}_{0}$ there exists a $\mathbf{k}$ primitive of $f, G$ with $\mathbf{D}^{\mathrm{k}} G=f$, that is a continuous function in a neighborhood of $\mathbf{x}=\mathbf{x}_{0}$ and satisfies

$$
G(\mathbf{x})=\frac{\gamma\left(\mathbf{x}-\mathbf{x}_{0}\right)^{\mathbf{k}}}{\mathbf{k} !}+o\left(\left|\mathbf{x}-\mathbf{x}_{0}\right|^{|\mathbf{k}|}\right), \quad \text { as } \mathbf{x} \rightarrow \mathbf{x}_{0} .
$$


It is important to observe that the distributional point values determine a distribution if they exists everywhere, that is, if $f \in \mathcal{D}^{\prime}\left(\mathbb{R}^{n}\right)$ is such that $f(\mathbf{x})=0$ distributionally $\forall \mathbf{x} \in \Omega$, where $\Omega$ is an open set, then $f=0$ in $\Omega[11,12]$.

There is a related but different notion of distributional point value, that of a symmetric value. We say that $f$ has the symmetric distributional value $\gamma$ at $\mathbf{x}=\mathbf{x}_{0}$, and write

$$
f_{\text {sym }}\left(\mathbf{x}_{0}\right)=\gamma, \text { distributionally, }
$$

if (2.2) holds for radial test functions. In the one variable case this means that $\left(f\left(x_{0}+x\right)+f\left(x_{0}-x\right)\right) / 2$ has the distributional value $\gamma$ at $x=0$. In several variables it means that $R(r)=\int_{\mathbb{S}} f\left(\mathbf{x}_{0}+r \omega\right) \mathrm{d} \sigma(\omega)$, when suitable extended to $\mathcal{D}^{\prime}(\mathbb{R})$, has the value $\gamma$ at $r=0$, where $\mathbb{S}$ is the unit sphere. A distribution like $\delta^{\prime}(x)$ has the symmetric value 0 at all points, so, in general, the symmetric distributional point values do not determine a distribution uniquely.

We shall follow $[3,5]$ for the notions related to Cesàro behavior of distributions. If $f \in \mathcal{D}^{\prime}\left(\mathbb{R}^{n}\right)$ and $\alpha \in \mathbb{R}$ is not a negative integer, we say that $f$ is bounded by $|\mathbf{x}|^{\alpha}$ in the Cesàro sense for $|\mathbf{x}|$ large, and write

$$
f(\mathbf{x})=O\left(|\mathbf{x}|^{\alpha}\right) \quad(\mathbf{C}), \quad \text { as }|\mathbf{x}| \rightarrow \infty,
$$

if there exists a multi-index $\mathbf{k} \in \mathbb{N}^{n}$ and a $\mathbf{k}$ primitive, $\mathbf{D}^{\mathbf{k}} G=f$, which is a function for $|\mathbf{x}|$ large and satisfies the ordinary order relation

$$
G(\mathbf{x})=O\left(|\mathbf{x}|^{\alpha+|\mathbf{k}|}\right), \quad \text { as }|\mathbf{x}| \rightarrow \infty .
$$

Naturally (2.6) will not hold for all primitives of $f$, and if it holds for $\mathbf{k}$ it will also hold for bigger multi-indices.

\section{ThE DisTRIBUTIONAL $\phi-$ TRANSFORM}

In this section we explain how we can extend to several variables the distributional $\phi$-transform introduced in [15]. Let $\phi \in \mathcal{D}\left(\mathbb{R}^{n}\right)$ be a fixed test function with

$$
\int_{\mathbb{R}^{n}} \phi(\mathbf{x}) \mathrm{d} \mathbf{x}=1
$$

If $f \in \mathcal{D}^{\prime}\left(\mathbb{R}^{n}\right)$ we introduce the function of $n+1$ variables $F=F_{\phi}\{f\}$ by the formula

$$
F(\mathbf{x}, t)=\langle f(\mathbf{x}+t \mathbf{y}), \phi(\mathbf{y})\rangle
$$


where $(\mathbf{x}, t) \in \mathbb{H}$, the half space $\mathbb{R}^{n} \times(0, \infty)$. Naturally the evaluation in (3.2) is with respect to the variable $\mathbf{y}$. We call $F$ the distributional $\phi$-transform of $f$. This transform also receives other names, such as the standard average with kernel $\phi[1]$. Whenever we consider $\phi$-transforms we assume that $\phi$ satisfies (3.1).

The definition of the $\phi$-transform tell us that if $f\left(\mathbf{x}_{0}\right)=\gamma$ then $F\left(\mathbf{x}_{0}, t\right) \rightarrow \gamma$ as $t \rightarrow 0^{+}$, but actually $F(\mathbf{x}, t) \rightarrow \gamma$ as $(\mathbf{x}, t) \rightarrow\left(\mathbf{x}_{0}, 0\right)$ in an angular or non-tangential fashion, that is if $\left|\mathbf{x}-\mathbf{x}_{0}\right| \leq M t$ for some $M>0$ (just replace $\phi(\mathbf{x})$ by $\phi(\mathbf{x}-M \omega)$ where $|\omega|=1$ ). On the other hand, if $f_{\text {sym }}\left(\mathbf{x}_{0}\right)=\gamma$ distributionally then $F\left(\mathbf{x}_{0}, t\right) \rightarrow \gamma$ as $t \rightarrow 0^{+}$whenever $\phi$ is radial, but in general $F(\mathbf{x}, t)$ does not approach $\gamma$ radially for general test functions and in general $F(\mathbf{x}, t)$ does not approach $\gamma$ in an angular fashion even if $\phi$ is radial.

We can also consider the $\phi$-transform if $\phi \in \mathcal{A}\left(\mathbb{R}^{n}\right)$ satisfies (3.1) and $f \in \mathcal{A}^{\prime}\left(\mathbb{R}^{n}\right)$, where $\mathcal{A}\left(\mathbb{R}^{n}\right)$ is a suitable space of test functions, such as $\mathcal{S}\left(\mathbb{R}^{n}\right)$ or $\mathcal{K}\left(\mathbb{R}^{n}\right)$.

We start with the distributional convergence of the $\phi$-transform.

Proposition 1. If $\phi \in \mathcal{D}\left(\mathbb{R}^{n}\right)$ and $f \in \mathcal{D}^{\prime}\left(\mathbb{R}^{n}\right)$, then

$$
\lim _{t \rightarrow 0^{+}} F(\mathbf{x}, t)=f(\mathbf{x}) \text {, }
$$

distributionally in the space $\mathcal{D}^{\prime}\left(\mathbb{R}^{n}\right)$, that is, if $\rho \in \mathcal{D}\left(\mathbb{R}^{n}\right)$ then

$$
\lim _{t \rightarrow 0^{+}}\langle F(\mathbf{x}, t), \rho(\mathbf{x})\rangle=\langle f(\mathbf{x}), \rho(\mathbf{x})\rangle .
$$

Proof. We have that

$$
\langle F(\mathbf{x}, t), \rho(\mathbf{x})\rangle=\langle\varrho(t \mathbf{y}), \phi(\mathbf{y})\rangle
$$

where

$$
\varrho(\mathbf{z})=\langle f(\mathbf{x}), \rho(\mathbf{x}-\mathbf{z})\rangle,
$$

is a smooth function of $\mathbf{z}$. The Łojasewicz point value $\varrho(\mathbf{0})$ exists and equals the ordinary value and thus

$$
\lim _{t \rightarrow 0^{+}}\langle\varrho(t \mathbf{y}), \phi(\mathbf{y})\rangle=\varrho(\mathbf{0})=\langle f(\mathbf{x}), \rho(\mathbf{x})\rangle,
$$

as required.

The result of the Proposition 1 also hold in other cases. In order to obtain those results we need some preliminary results. Recall that an asymptotic order relation is strong if it remains valid after differentiation of any order. 
Proposition 2. Let $f \in \mathcal{E}^{\prime}\left(\mathbb{R}^{n}\right)$ be a distribution with compact support $K$. Let $\phi \in \mathcal{E}\left(\mathbb{R}^{n}\right)$ be a test function that satisfies (3.1) and

$$
\phi(\mathbf{x})=O\left(|\mathbf{x}|^{\beta}\right), \quad \text { strongly as }|\mathbf{x}| \rightarrow \infty,
$$

where $\beta<-n$. Then

$$
\lim _{t \rightarrow 0^{+}} F(\mathbf{x}, t)=0,
$$

uniformly on compacts of $\mathbb{R}^{n} \backslash K$.

Proof. There exist a constant $M>0$ and $q \in \mathbb{N}$ such that

$$
|\langle f(\mathbf{y}), \rho(\mathbf{y})\rangle| \leq M \sum_{|\mathbf{j}|=0}^{q}\left\|\mathbf{D}^{\mathbf{j}} \rho\right\|_{K, \infty} \quad \forall \rho \in \mathcal{E}\left(\mathbb{R}^{n}\right),
$$

where $\|\rho\|_{K, \infty}=\sup \{|\rho(\mathbf{x})|: \mathbf{x} \in K\}$. There exist $r_{0}>0$ and constants $M_{\mathbf{j}}>0$ such that $\left|\mathbf{D}^{\mathbf{j}} \phi(\mathbf{x})\right| \leq M_{\mathbf{j}}|\mathbf{x}|^{\beta-|\mathbf{j}|}$ for $|\mathbf{x}| \geq r_{0}$ and $|\mathbf{j}| \leq q$. Let $L$ be a compact subset of $\mathbb{R}^{n} \backslash K$, and let $t_{0}>0$ be such that if $0<t \leq t_{0}$ then $t^{-1}|\mathbf{x}-\mathbf{y}| \geq r_{0}$ for all $\mathbf{x} \in L, \mathbf{y} \in K$. Then, since

$$
F(\mathbf{x}, t)=t^{-n}\left\langle f(\mathbf{y}), \phi\left(t^{-1}(\mathbf{y}-\mathbf{x})\right)\right\rangle,
$$

it follows that for $0<t \leq t_{0}$,

$$
|F(\mathbf{x}, t)| \leq M_{2} t^{-n-\beta}, \quad \forall \mathbf{x} \in L,
$$

where $M_{2}=M \sum_{|\mathbf{j}|=0}^{q} M_{\mathbf{j}}$ is a constant. Since $-\beta-n>0$, we obtain that (3.9) holds uniformly on $\mathbf{x} \in L$.

The definition of the Łojasiewicz point value is that if $f \in \mathcal{D}^{\prime}\left(\mathbb{R}^{n}\right)$ then $f\left(\mathbf{x}_{0}\right)=\gamma$ distributionally if

$$
\lim _{\varepsilon \rightarrow 0}\left\langle f\left(\mathbf{x}_{0}+\varepsilon \mathbf{x}\right), \phi(\mathbf{x})\right\rangle=\gamma \int_{\mathbb{R}^{n}} \phi(\mathbf{x}) \mathrm{d} \mathbf{x}
$$

whenever $\phi \in \mathcal{D}\left(\mathbb{R}^{n}\right)$. If $f$ belongs to a smaller class of distributions, then $\left\langle f\left(\mathbf{x}_{0}+\varepsilon \mathbf{x}\right), \phi(\mathbf{x})\right\rangle$ will be defined for test functions of a larger class, not only for those of $\mathcal{D}\left(\mathbb{R}^{n}\right)$, and one may ask whether (3.13) remains true in that case. There are cases where (3.13) is not true, for instance if $f \in \mathcal{E}^{\prime}(\mathbb{R})$ sometimes there are $\phi \in \mathcal{E}(\mathbb{R})$ that do not satisfy (3.13) [4]. However, it was shown in [4] that in the one variable case, (3.13) holds if $f\left(x_{0}\right)=\gamma$ distributionally and the following conditions are satisfied:

$$
\begin{gathered}
f(x)=O\left(|x|^{\alpha}\right) \quad(\mathrm{C}), \text { as }|x| \rightarrow \infty, \\
\phi(x)=O\left(|x|^{\beta}\right), \quad \text { strongly as }|x| \rightarrow \infty,
\end{gathered}
$$




$$
\alpha+\beta<-1, \quad \beta<-1 .
$$

In particular, (3.13) is valid when $f \in \mathcal{S}^{\prime}(\mathbb{R})$ and $\phi \in \mathcal{S}(\mathbb{R})[4,13,17]$. Actually a corresponding result is valid in several variables, and the proof is basically the same.

Proposition 3. Let $f \in \mathcal{D}^{\prime}\left(\mathbb{R}^{n}\right)$ with $f\left(\mathbf{x}_{0}\right)=\gamma$ distributionally. Let $\phi \in \mathcal{E}\left(\mathbb{R}^{n}\right)$. Suppose that

$$
\begin{gathered}
f(\mathbf{x})=O\left(|\mathbf{x}|^{\alpha}\right) \quad(C), \text { as }|\mathbf{x}| \rightarrow \infty, \\
\phi(\mathbf{x})=O\left(|\mathbf{x}|^{\beta}\right), \quad \text { strongly as }|\mathbf{x}| \rightarrow \infty,
\end{gathered}
$$

where

$$
\alpha+\beta<-n, \quad \beta<-n .
$$

Then

$$
\lim _{\varepsilon \rightarrow 0}\left\langle f\left(\mathbf{x}_{0}+\varepsilon \mathbf{x}\right), \phi(\mathbf{x})\right\rangle=\gamma \int_{\mathbb{R}^{n}} \phi(\mathbf{x}) \mathrm{d} \mathbf{x} .
$$

Proof. Suppose that $\mathbf{x}_{0}=\mathbf{0}$. There exists a muti-index $\mathbf{k}$ and two primitives of $f, \mathbf{D}^{\mathbf{k}} G_{1}=\mathbf{D}^{\mathbf{k}} G_{2}=f$ such that

$$
\begin{gathered}
G_{1}(\mathbf{x})=O\left(|\mathbf{x}|^{\alpha+|\mathbf{k}|}\right), \quad \text { as }|\mathbf{x}| \rightarrow \infty, \\
G_{2}(\mathbf{x})=\frac{\gamma \mathbf{x}^{\mathbf{k}}}{\mathbf{k} !}+o\left(|\mathbf{x}|^{|\mathbf{k}|}\right), \quad \text { as }|\mathbf{x}| \rightarrow 0 .
\end{gathered}
$$

Hence we can combine them into a single function $G$ that satisfies

$$
\begin{aligned}
\left|G(\mathbf{x})-\frac{\gamma \mathbf{x}^{\mathbf{k}}}{\mathbf{k} !}\right| & \leq M|\mathbf{x}|^{|\mathbf{k}|}, \text { for }|\mathbf{x}| \leq 1, \\
|G(\mathbf{x})| & \leq M|\mathbf{x}|^{\alpha+|\mathbf{k}|}, \text { for }|\mathbf{x}| \geq 1,
\end{aligned}
$$

and

$$
f=g+\mathbf{D}^{\mathrm{k}} G,
$$

where $g$ has compact support and $\mathbf{x}_{0} \notin \operatorname{supp} g$. Then (3.20) holds for $g$, because of the Proposition 2, and it holds if $\phi$ has compact support. Therefore it is enough to prove (3.20) if $f=\mathbf{D}^{\mathrm{k}} G$ and $\operatorname{supp} \phi \subset\{\mathbf{x}:|\mathbf{x}| \geq 1\}$; but in this case we may use the Lebesgue 
bounded convergence theorem to obtain

$$
\begin{aligned}
\lim _{\varepsilon \rightarrow 0}\langle f(\varepsilon \mathbf{x}), \phi(\mathbf{x})\rangle & =\lim _{\varepsilon \rightarrow 0}(-1)^{|\mathbf{k}|} \varepsilon^{-|\mathbf{k}|} \int_{\mathbb{R}^{n}} G(\varepsilon \mathbf{x}) \mathbf{D}^{\mathbf{k}} \phi(\mathbf{x}) \mathrm{d} \mathbf{x} \\
& =\frac{(-1)^{|\mathbf{k}|} \gamma}{\mathbf{k} !} \int_{\mathbb{R}^{n}} \mathbf{x}^{\mathbf{k}} \mathbf{D}^{\mathbf{k}} \phi(\mathbf{x}) \mathrm{d} \mathbf{x} \\
& =\gamma \int_{\mathbb{R}^{n}} \phi(\mathbf{x}) \mathrm{d} \mathbf{x}
\end{aligned}
$$

as required.

In particular, (3.20) holds if $f \in \mathcal{S}^{\prime}\left(\mathbb{R}^{n}\right)$ and $\phi \in \mathcal{S}\left(\mathbb{R}^{n}\right)$.

Using the same argument as in the last proof we can prove that if $f(\mathbf{x})=0$ for $\mathbf{x} \in \Omega$, an open set, and the conditions (3.17), (3.18), and (3.19) are satisfied, then the convergence in (3.20) is uniform on compacts of $\Omega$.

We can now extend the distributional convergence of the $\phi$-transform, Proposition 1, to other cases.

Proposition 4. If $\phi \in \mathcal{E}\left(\mathbb{R}^{n}\right)$ and $f \in \mathcal{E}^{\prime}\left(\mathbb{R}^{n}\right)$ satisfy the conditions (3.17), (3.18), and (3.19), then

$$
\lim _{t \rightarrow 0^{+}} F(\mathbf{x}, t)=f(\mathbf{x}),
$$

distributionally in the space $\mathcal{D}^{\prime}\left(\mathbb{R}^{n}\right)$, that is, if $\rho \in \mathcal{D}\left(\mathbb{R}^{n}\right)$ then

$$
\lim _{t \rightarrow 0^{+}}\langle F(\mathbf{x}, t), \rho(\mathbf{x})\rangle=\langle f(\mathbf{x}), \rho(\mathbf{x})\rangle .
$$

In particular, distributional convergence, (3.24), holds if $\phi \in \mathcal{S}\left(\mathbb{R}^{n}\right)$ and $f \in \mathcal{S}^{\prime}\left(\mathbb{R}^{n}\right)$.

Proof. We proceed as in the proof of the Proposition 1 by observing that $\langle F(\mathbf{x}, t), \rho(\mathbf{x})\rangle=\langle\varrho(t \mathbf{y}), \phi(\mathbf{y})\rangle$, where $\varrho(\mathbf{z})=\langle f(\mathbf{x}), \rho(\mathbf{x}-\mathbf{z})\rangle$. Next we observe that $\varrho$ is a smooth function, and that it satisfies $\varrho(\mathbf{x})=O\left(|\mathbf{x}|^{\alpha}\right)(\mathrm{C})$, as $|\mathbf{x}| \rightarrow \infty$. Indeed, there exists a multi-index $\mathbf{k}$ and a primitive of $f$ of that order, $\mathbf{D}^{\mathbf{k}} G=f$, which is an ordinary function for large arguments and satisfies $|G(\mathbf{x})|=O\left(|\mathbf{x}|^{|\mathbf{k}|+\alpha}\right)$ as $|\mathbf{x}| \rightarrow \infty$. We have then that

$$
\begin{aligned}
\varrho(\mathbf{z}) & =\left\langle\mathbf{D}_{\mathbf{x}}^{\mathbf{k}} G(\mathbf{x}), \rho(\mathbf{x}-\mathbf{z})\right\rangle \\
& =\mathbf{D}_{\mathbf{z}}^{\mathbf{k}}\langle G(\mathbf{x}), \rho(\mathbf{x}-\mathbf{z})\rangle,
\end{aligned}
$$

and $\langle G(\mathbf{x}), \rho(\mathbf{x}-\mathbf{z})\rangle=\int_{\operatorname{supp} \rho} G(\mathbf{x}+\mathbf{z}) \rho(\mathbf{x}) \mathrm{d} \mathbf{x}=O\left(|\mathbf{z}|^{|\mathbf{k}|+\alpha}\right)$ as $|\mathbf{z}| \rightarrow$ $\infty$, since supp $\rho$ is compact. Hence the Proposition 3 allows us to obtain that $\lim _{t \rightarrow 0^{+}}\langle\varrho(t \mathbf{y}), \phi(\mathbf{y})\rangle=\varrho(\mathbf{0})=\langle f(\mathbf{x}), \rho(\mathbf{x})\rangle$. 
Observe also if $\phi \in \mathcal{E}\left(\mathbb{R}^{n}\right)$ and $f \in \mathcal{E}^{\prime}\left(\mathbb{R}^{n}\right)$ satisfy the conditions (3.17), (3.18), and (3.19), then when the distributional point value $f\left(\mathbf{x}_{0}\right)$ exists then $F(\mathbf{x}, t) \rightarrow f\left(\mathbf{x}_{0}\right)$ as $(\mathbf{x}, t) \rightarrow\left(\mathbf{x}_{0}, 0\right)$ in an angular fashion, while if the distributional symmetric value $f_{\text {sym }}\left(\mathbf{x}_{0}\right)$ exists and $\phi$ is radial then $F\left(\mathbf{x}_{0}, t\right) \rightarrow f\left(\mathbf{x}_{0}\right)$ as $t \rightarrow 0^{+}$.

\section{Summability Methods}

In this section we explain several methods of summability that one can use in connection with the Fourier inversion formula. We start with the $\psi$-summability.

4.1. The $\psi$-summability. Let $\psi \in \mathcal{S}\left(\mathbb{R}^{n}\right)$ be any function with $\psi(\mathbf{0})=1$. If $g \in \mathcal{S}^{\prime}\left(\mathbb{R}^{n}\right)$ and $\rho$ is a smooth function in $\mathbb{R}^{n}$ with $\rho g \in \mathcal{S}^{\prime}\left(\mathbb{R}^{n}\right)$, then the evaluation

$$
\langle g(\mathbf{x}), \rho(\mathbf{x})\rangle,
$$

is not defined, in general, because $\rho$ may not belong to $\mathcal{S}\left(\mathbb{R}^{n}\right)$. However, if $\varepsilon>0$, the evaluation

$$
G(\varepsilon)=\langle g(\mathbf{x}), \rho(\mathbf{x}) \psi(\varepsilon \mathbf{x})\rangle,
$$

is well-defined. If

$$
\lim _{\varepsilon \rightarrow 0} G(\varepsilon)=S,
$$

exists, then we say that the evaluation $\langle g(\mathbf{x}), \rho(\mathbf{x})\rangle$ is $\psi$-summable to $S$, and write

$$
\langle g(\mathbf{x}), \rho(\mathbf{x})\rangle=S \quad(\psi) .
$$

When $g$ is locally integrable, then (4.4) can be written as

$$
\int_{\mathbb{R}^{n}} g(\mathbf{x}) \rho(\mathbf{x}) \mathrm{d} \mathbf{x}=S \quad(\psi),
$$

while if $g(\mathbf{x})=\sum_{n=1}^{\infty} a_{n} \delta\left(\mathbf{x}-\mathbf{b}_{n}\right)$, then (4.4) becomes

$$
\sum_{n=1}^{\infty} a_{n} \rho\left(\mathbf{b}_{n}\right)=S \quad(\psi) .
$$

In particular, if $\psi(\mathbf{x})=e^{-|\mathbf{x}|^{2}}$ then the $(\psi)$ summability becomes the Gauss-Weierestrass summability; we may write $\langle g(\mathbf{x}), \rho(\mathbf{x})\rangle(\mathrm{G}-\mathrm{W})$ in this case. 
Proposition 5. Let $\psi \in \mathcal{S}\left(\mathbb{R}^{n}\right)$ with $\psi(\mathbf{0})=1$. Let $f \in \mathcal{S}^{\prime}\left(\mathbb{R}^{n}\right)$. Then

$$
f(\mathbf{x})=\frac{1}{(2 \pi)^{n}}\left\langle\widehat{f}(\mathbf{u}), e^{-i \mathbf{u} \bullet \mathbf{x}}\right\rangle \quad(\psi)
$$

distributionally in the space $\mathcal{D}^{\prime}\left(\mathbb{R}^{n}\right)$, that is, $\forall \rho \in \mathcal{D}\left(\mathbb{R}^{n}\right)$,

$$
\lim _{\varepsilon \rightarrow 0^{+}}\left\langle\frac{1}{(2 \pi)^{n}}\left\langle\widehat{f}(\mathbf{u}), e^{-i \mathbf{u} \mathbf{x}} \psi(\varepsilon \mathbf{u})\right\rangle, \rho(\mathbf{x})\right\rangle=\langle f(\mathbf{x}), \rho(\mathbf{x})\rangle .
$$

Moreover, relation (4.7) holds pointwise at any point $\mathbf{x}$ where the distributional point value $f(\mathbf{x})$ exists.

Proof. The result follows immediately from the Propositions 3 and 4 because

$$
\frac{1}{(2 \pi)^{n}}\left\langle\widehat{f}(\mathbf{u}), e^{-i \mathbf{u} \bullet \mathbf{x}} \psi(\varepsilon \mathbf{u})\right\rangle=F(\mathbf{x}, \varepsilon)
$$

where $F$ is the $\phi$-transform of $f$ for $\phi(\mathbf{x})=(2 \pi)^{-n} \widehat{\psi}(\mathbf{x})$.

Observe, in particular, that the Fourier inversion formula is always valid distributionally, in the space $\mathcal{D}^{\prime}\left(\mathbb{R}^{n}\right)$, in the Gauss-Weierestrass summability sense for any tempered distribution.

We also have pointwise convergence at all points where the symmetric point value exists, provided that $\psi$ is radial.

Proposition 6. Let $\psi \in \mathcal{S}\left(\mathbb{R}^{n}\right)$ be a radial test function with $\psi(\mathbf{0})=$ 1. Let $f \in \mathcal{S}^{\prime}\left(\mathbb{R}^{n}\right)$. Let $\mathbf{x}_{0} \in \mathbb{R}^{n}$ be a point where the distributional symmetric value $f_{\mathrm{sym}}\left(\mathbf{x}_{0}\right)$ exists. Then

$$
f_{\mathrm{sym}}\left(\mathbf{x}_{0}\right)=\frac{1}{(2 \pi)^{n}}\left\langle\widehat{f}(\mathbf{u}), e^{-i \mathbf{u} \bullet \mathbf{x}_{0}}\right\rangle \quad(\psi)
$$

4.2. Abel summability. The Abel method of summability follows by taking $\psi(\mathbf{x})=e^{-|\mathbf{x}|}$ in the $(\psi)$ summability procedure:

$$
\langle g(\mathbf{x}), \rho(\mathbf{x})\rangle=S \quad(\mathrm{~A}) .
$$

if

$$
\lim _{\varepsilon \rightarrow 0^{+}}\left\langle g(\mathbf{x}), \rho(\mathbf{x}) e^{-\varepsilon|\mathbf{x}|}\right\rangle=S .
$$

There is an obvious problem in the application of this method, namely, the function $e^{-|\mathbf{x}|}$ does not belong to $\mathcal{S}\left(\mathbb{R}^{n}\right)$ since it is not differentiable at $\mathbf{x}=\mathbf{0}$. It is fair to say, however, that $e^{-|\mathbf{x}|}$ does have the behavior of the space $\mathcal{S}\left(\mathbb{R}^{n}\right)$ as $|\mathbf{x}| \rightarrow \infty$. If $g$ satisfies certain conditions near $\mathbf{x}=\mathbf{0}$, then $\left\langle g(\mathbf{x}), \rho(\mathbf{x}) e^{-\varepsilon|\mathbf{x}|}\right\rangle$ can be computed, for instance, if $g$ is a locally integrable function in a neighborhood of $\mathbf{x}=\mathbf{0}$, 
or more generally if it is a Radon signed measure in such a neighborhood.

We can consider Abel means for general $g$ if we accept that in some cases these means are not unique. Indeed, let $e(g)$ be an extension of $g \in \mathcal{S}^{\prime}\left(\mathbb{R}^{n}\right)$ to the dual space $(\mathcal{X} \widehat{\otimes} \mathcal{D}(\mathbb{S}))^{\prime}$, where we use polar coordinates $\mathbf{x}=r \omega, r \geq 0, \omega \in \mathbb{S}$, and where $\mathcal{X}$ is the space of restrictions of functions $\rho(r)$ for $\rho \in \mathcal{S}(\mathbb{R})$ to $[0, \infty)$. Then $\rho(\mathbf{x}) e^{-\varepsilon|\mathbf{x}|}$ belongs to $\mathcal{X} \widehat{\otimes} \mathcal{D}(\mathbb{S})$ and thus we can consider the Abel means $G(\varepsilon)=$ $\left\langle e(g)(\mathbf{x}), \rho(\mathbf{x}) e^{-\varepsilon|\mathbf{x}|}\right\rangle$, and its limit as $\varepsilon \searrow 0$ instead of (4.12). Some $g$ have canonical extensions $e(g)$, but in general $e(g)$ is not uniquely defined.

If we use Abel summability in the Fourier inversion formula, we obtain the means

$$
U(\mathbf{x}, t)=\frac{1}{(2 \pi)^{n}}\left\langle e(\widehat{f})(\mathbf{u}), e^{-i \mathbf{u} \mathbf{0} \mathbf{x}-t|\mathbf{u}|}\right\rangle,
$$

which is harmonic in $\mathbb{H}: U_{t t}+\sum_{j=1}^{n} U_{x_{j} x_{j}}=0$. A similar analysis to that of Proposition 5 yields

$$
\lim _{t \rightarrow 0^{+}} U(\mathbf{x}, t)=f(\mathbf{x}) .
$$

We also observe that for a fixed $t>0$ the function $U(\mathbf{x}, t)$ belongs to $\mathcal{S}^{\prime}\left(\mathbb{R}^{n}\right)$.

We can thus say that the Abel means in the Fourier inversion formula of a tempered distribution $f \in \mathcal{S}^{\prime}\left(\mathbb{R}^{n}\right)$ are those harmonic functions in $\mathbb{H}$ with these properties. Functions like $U(\mathbf{x}, t)=t$ or $U(\mathbf{x}, t)=3 x_{j}^{2} t-$ $t^{3}$ are Abel means of $f=0$, and thus the source of non-uniqueness.

If $f \in \mathcal{E}^{\prime}\left(\mathbb{R}^{n}\right)$, or more generally if $f(\mathbf{x})=O(1)$ (C) as $|\mathbf{x}| \rightarrow \infty$, then one can define a canonical Abel mean for the Fourier inversion formula as

$$
U(\mathbf{x}, t)=c_{n}\left\langle f(\mathbf{y}), \frac{t}{\left(t^{2}+\|\mathbf{x}-\mathbf{y}\|^{2}\right)^{\frac{n+1}{2}}}\right\rangle,
$$

where

$$
c_{n}=\frac{\Gamma\left(\frac{n+1}{2}\right)}{\pi^{\frac{n+1}{2}}}=\left(\int_{\mathbb{R}^{n}} \frac{\mathrm{d} \mathbf{y}}{\left(1+\|\mathbf{y}\|^{2}\right)^{\frac{n+1}{2}}}\right)^{-1},
$$

and where the kernel in (4.15) is the Poisson kernel for $\mathbb{H}$. In this case $U(\mathbf{x}, t)$ is the $\phi$-transform of $f$ for $\phi(\mathbf{y})=c_{n}\left(1+\|\mathbf{y}\|^{2}\right)^{-\frac{n+1}{2}}$.

Observe that if the distributional symmetric value $f_{\text {sym }}\left(\mathbf{x}_{0}\right)$ exists then for any Abel mean $U(\mathbf{x}, t)$ we have that $U\left(\mathbf{x}_{0}, t\right) \rightarrow f_{\text {sym }}\left(\mathbf{x}_{0}\right)$, 
that is,

$$
f_{\mathrm{sym}}\left(\mathbf{x}_{0}\right)=\frac{1}{(2 \pi)^{n}}\left\langle\widehat{f}(\mathbf{u}), e^{-i \mathbf{u} \mathbf{0} \mathbf{x}_{0}}\right\rangle
$$

4.3. Cesàro summability. We can also consider Cesàro summability by spherical means [5, Section 6.8]. Summability by spherical means can actually be reduced to summability in one variable since using polar coordinates, $\mathbf{x}=r \omega, r \geq 0, \omega \in \mathbb{S}$, we obtain

$$
\left\langle f(\mathbf{x}), 1_{\mathbf{x}}\right\rangle=\left\langle F(r), r^{n-1}\right\rangle \quad(\mathrm{C}),
$$

where

$$
F(r)=\left\langle f(r \omega), 1_{\omega}\right\rangle_{\mathcal{D}^{\prime}(\mathbb{S}) \times \mathcal{D}(\mathbb{S})} .
$$

The distribution $F$ is not uniquely defined at $r=0$, however we can always write $f=f_{1}+f_{2}$, where $f_{1}$ has compact support and where $\mathbf{0} \notin \operatorname{supp} f_{2}$. The evaluation $\left\langle f_{1}(\mathbf{x}), \phi(\mathbf{x})\right\rangle$ is well-defined for any $\phi \in$ $\mathcal{E}\left(\mathbb{R}^{n}\right)$, so we need to consider only the case when $f=f_{2}$ satisfies that supp $f \subset\{\mathbf{x}:|\mathbf{x}| \geq a\}$ for some $a>0$. Then $F$ will be uniquely defined if we require that $\operatorname{supp} F \subset[a, \infty)$.

We now explain when $\langle f(\mathbf{x}), \phi(\mathbf{x})\rangle$ is Cesàro summable by spherical means of order $N$,

$$
\langle f(\mathbf{x}), \phi(\mathbf{x})\rangle=L \quad(\mathrm{C}, N)_{\mathrm{r}} .
$$

If $\phi=1$ the $(\mathrm{C}, N)_{\mathrm{r}}$ summability means that the one-variable evaluation

$$
\left\langle F(r), r^{n-1}\right\rangle=L \quad(\mathrm{C}, N),
$$

exists in the $(\mathrm{C}, N)$ sense. For a general $\phi$ it means that $\left\langle\phi(\mathbf{x}) f(\mathbf{x}), 1_{\mathbf{x}}\right\rangle$ $=L(\mathrm{C}, N)_{\mathrm{r}}$. The notation $(\mathrm{C})_{\mathrm{r}}$ is used for Cesàro summability by spherical means, namely when there exists some $N$ such that the evaluation is $(\mathrm{C}, N)_{\mathrm{r}}$.

Observe that the $(\mathrm{C}, N)_{\mathrm{r}}$ summability corresponds to the case where

$$
\psi_{N}(\mathbf{x})=H(1-|\mathbf{x}|) \frac{(1-|\mathbf{x}|)^{N}}{N !}
$$

in the $\psi$-summability. Here $H$ is the Heaviside function.

If $f \in \mathcal{K}^{\prime}\left(\mathbb{R}^{n}\right)$ and $\phi \in \mathcal{K}\left(\mathbb{R}^{n}\right)$, then the evaluation $\langle f, \phi\rangle$ exists in the $(\mathrm{C})_{\mathrm{r}}$ sense, that is, it exists $(\mathrm{C}, N)_{\text {r }}$ for some $N$. The value of $N$ depends on $\phi$ in this case: Consider the example where $f(x)=e^{i x}$ and $\phi(x)=x^{n}$. On the other hand, if $f \in \mathcal{S}^{\prime}\left(\mathbb{R}^{n}\right)$ and $\phi \in \mathcal{S}\left(\mathbb{R}^{n}\right)$ then the evaluation $\langle f, \phi\rangle$ also exists $(\mathrm{C})_{\mathrm{r}}$ since $\langle f, \phi\rangle=\langle\phi f, 1\rangle$, and $\phi f \in \mathcal{K}^{\prime}\left(\mathbb{R}^{n}\right)$, but now if $f \in \mathcal{S}^{\prime}\left(\mathbb{R}^{n}\right)$ is fixed then there exists $N$ such that $\langle f, \phi\rangle$ exists $(\mathrm{C}, N)_{\mathrm{r}}$ for all test functions $\phi \in \mathcal{S}\left(\mathbb{R}^{n}\right)$. 
The Cesàro means of the Fourier inversion formula will converge distributionally, as in the case of the Abel means and the $(\psi)$ means, but this happens if $N$ is large.

Proposition 7. Let $f \in \mathcal{S}^{\prime}\left(\mathbb{R}^{n}\right)$. Then there exists $N$ such that

$$
f(\mathbf{x})=\frac{1}{(2 \pi)^{n}}\left\langle\widehat{f}(\mathbf{u}), e^{-i \mathbf{u} \mathbf{x}}\right\rangle \quad(C, N)_{r},
$$

distributionally in the space $\mathcal{S}^{\prime}\left(\mathbb{R}^{n}\right)$, in the sense that for each $\rho \in$ $\mathcal{S}\left(\mathbb{R}^{n}\right)$

$$
\lim _{\varepsilon \rightarrow 0^{+}}\left\langle\frac{1}{(2 \pi)^{n}}\left\langle\widehat{f}(\mathbf{u}), e^{-i \mathbf{u} \mathbf{0}} \psi_{N}(\varepsilon \mathbf{u})\right\rangle, \rho(\mathbf{x})\right\rangle=\langle f(\mathbf{x}), \rho(\mathbf{x})\rangle \quad(C, N)_{r} .
$$

Proof. Indeed,

$$
\left\langle\frac{1}{(2 \pi)^{n}}\left\langle\widehat{f}(\mathbf{u}), e^{-i \mathbf{u} \mathbf{x}}\right\rangle, \rho(\mathbf{x})\right\rangle=\frac{1}{(2 \pi)^{n}}\langle\widehat{f}(\mathbf{u}), \widehat{\rho}(-\mathbf{u})\rangle,
$$

and there exists $N$ such that the evaluation $\langle\widehat{f}, \phi\rangle$ exists $(\mathrm{C}, N)_{\text {r }}$ for all test functions $\phi \in \mathcal{S}\left(\mathbb{R}^{n}\right)$, in particular for $\phi(\mathbf{u})=\widehat{\rho}(-\mathbf{u})$. But since $(2 \pi)^{-n}\langle\widehat{f}(\mathbf{u}), \widehat{\rho}(-\mathbf{u})\rangle=\langle f(\mathbf{x}), \rho(\mathbf{x})\rangle,(4.24)$ is obtained.

It is interesting to observe if $f \in \mathcal{E}^{\prime}\left(\mathbb{R}^{n}\right)$ then there is no need to use Cesàro summability in (4.23), that is, we actually get convergence of the spherical means. Similarly, if $f$ is periodic of periods in $\prod_{j=1}^{n} \tau_{j} \mathbb{Z}$, so that its Fourier transform is concentrated on a discrete set, and the Fourier inversion formula is the Fourier series, then we also get convergence. However, for a general $f \in \mathcal{S}^{\prime}\left(\mathbb{R}^{n}\right)$ there is a value $N$ for which (4.23) holds, but the spherical means are not (C,M) summable if $M<N$.

When the distributional symmetric value $f_{\text {sym }}\left(\mathbf{x}_{0}\right)$ exists then (1.4) implies that we have pointwise Cesàro summability,

$$
f_{\mathrm{sym}}\left(\mathbf{x}_{0}\right)=\frac{1}{(2 \pi)^{n}}\left\langle\widehat{f}(\mathbf{u}), e^{-i \mathbf{u} \bullet \mathbf{x}_{0}}\right\rangle \quad(\mathrm{C}, N)_{\mathrm{r}},
$$

if $N$ is large.

\section{Continuity}

If $U(\mathbf{x}, t)$ is harmonic in $\mathbb{H}$, with distributional boundary value $f(\mathbf{x})=$ $U\left(\mathbf{x}, 0^{+}\right) \in \mathcal{S}^{\prime}\left(\mathbb{R}^{n}\right)$, and $f$ is continuous in an open set $\Omega \subset \mathbb{R}^{n}$, then it is well-known that actually $U(\mathbf{x}, t)$ can be extended as a continuous function to $\mathbb{H} \cup(\Omega \times\{0\})$, and consequently, $U(\mathbf{x}, t) \rightarrow f(\mathbf{x})$ 
uniformly on compacts of $\Omega$. In fact, this is a general result for the $\phi$-transform.

Proposition 8. Let $f \in \mathcal{D}^{\prime}\left(\mathbb{R}^{n}\right)$ and let $F(\mathbf{x}, t)$ be its $\phi$-transform. Suppose that $\phi \in \mathcal{D}\left(\mathbb{R}^{n}\right)$ or that (3.17), (3.18), and (3.19) are satisfied. If $f$ is an ordinary bounded function in a neighborhood of a point $\mathbf{x}_{0}$ and that function is continuous at $\mathbf{x}=\mathbf{x}_{0}$ then

$$
\lim _{(\mathbf{x}, t) \rightarrow\left(\mathbf{x}_{0}, 0\right)} F(\mathbf{x}, t)=f\left(\mathbf{x}_{0}\right),
$$

so that $F$ can be extended as a continuous function to $\mathbb{H} \cup\left(\left\{\mathbf{x}_{0}\right\} \times\{0\}\right)$.

Proof. The results of Section 3 show that (5.1) holds if $\mathbf{x}_{0} \in \mathbb{R}^{n} \backslash \operatorname{supp} f$. Hence, it is enough to prove (5.1) when $f$ is an ordinary bounded function with compact support. Let $\varepsilon>0$, and let $B$ be an open neighborhood of $\mathbf{x}_{0}$, with compact closure, such that $\left|f(\mathbf{y})-f\left(\mathbf{x}_{0}\right)\right|<$ $\varepsilon$ for $\mathbf{y} \in B$. Write $F(\mathbf{x}, t)-f\left(\mathbf{x}_{0}\right)=G_{1}(\mathbf{x}, t)+G_{2}(\mathbf{x}, t)$, where

$$
\begin{aligned}
& G_{1}(\mathbf{x}, t)=t^{-n} \int_{B}\left(f(\mathbf{y})-f\left(\mathbf{x}_{0}\right)\right) \phi\left(t^{-1}(\mathbf{y}-\mathbf{x})\right) \mathrm{d} \mathbf{y} \\
& G_{2}(\mathbf{x}, t)=t^{-n} \int_{\mathbb{R}^{n} \backslash B}\left(f(\mathbf{y})-f\left(\mathbf{x}_{0}\right)\right) \phi\left(t^{-1}(\mathbf{y}-\mathbf{x})\right) \mathrm{d} \mathbf{y} .
\end{aligned}
$$

Then $G_{2}(\mathbf{x}, t) \rightarrow 0$ as $t \rightarrow 0$ uniformly on compacts of $B$, while

$$
\left|G_{1}(\mathbf{x}, t)\right| \leq \varepsilon \int_{\mathbb{R}^{n}}|\phi(\mathbf{y})| \mathrm{d} \mathbf{y}
$$

and (5.1) follows.

Observe that if the conditions of the Proposition 8 are satisfied and $f\left(\mathbf{x}_{0}\right)=\gamma$ distributionally then $F(\mathbf{x}, t) \rightarrow \gamma$ as $(\mathbf{x}, t) \rightarrow\left(\mathbf{x}_{0}, 0\right)$ in a nontangential fashion, while if the distributional symmetric value exists, $f_{\text {sym }}\left(\mathbf{x}_{0}\right)=\gamma$, and $\phi$ is radial then $F\left(\mathbf{x}_{0}, t\right) \rightarrow \gamma$ as $t \rightarrow 0^{+}$. According to Proposition 8 if $f$ is continuous at $\mathbf{x}=\mathbf{x}_{0}$ then $F(\mathbf{x}, t) \rightarrow \gamma$ as $(\mathbf{x}, t) \rightarrow\left(\mathbf{x}_{0}, 0\right)$ in an unrestricted fashion.

Proposition 9. Let $f \in \mathcal{D}^{\prime}\left(\mathbb{R}^{n}\right)$ and let $F(\mathbf{x}, t)$ be its $\phi$-transform. Suppose that $\phi \in \mathcal{D}\left(\mathbb{R}^{n}\right)$ or that (3.17), (3.18), and (3.19) are satisfied. If $f$ is a continuous function in an open set $\Omega \subset \mathbb{R}^{n}$ then $F$ can be extended as a continuous function to $\mathbb{H} \cup(\Omega \times\{0\})$, and $F(\mathbf{x}, t) \rightarrow f(\mathbf{x})$ uniformly on compacts of $\Omega$. Conversely, if $F(\mathbf{x}, t) \rightarrow f(\mathbf{x})$ uniformly on compacts of $\Omega$, then $f$ is a continuous function in $\Omega$.

Proof. The direct part follows immediately from the previous proposition, while the converse result follows because uniform convergence on compacts implies distributional convergence. 
In particular, we have the following result for summability of the Fourier inversion formula.

Corollary 1. Let $f \in \mathcal{S}^{\prime}\left(\mathbb{R}^{n}\right)$. If $f$ is a continuous function in an open set $\Omega \subset \mathbb{R}^{n}$ then the $\psi$ means, for any $\psi \in \mathcal{S}\left(\mathbb{R}^{n}\right)$, any Abel means, or the Cesàro means of large order converge to $f$ uniformly on compacts of $\Omega$ :

$$
f(\mathbf{x})=\frac{1}{(2 \pi)^{n}}\left\langle\widehat{f}(\mathbf{u}), e^{-i \mathbf{u} \bullet \mathbf{x}}\right\rangle \quad(\mathrm{T})
$$

uniformly on $\mathbf{x} \in K, K$ a compact subset of $\Omega$, for $(\mathrm{T})=(\psi),(A)$, or $(C, N)_{r}$ for $N$ large. Conversely, if (5.5) holds uniformly on compacts of $\Omega$ then $f$ is a continuous function on $\Omega$.

\section{The SUPPort OF A Distribution}

We now show how we can obtain a characterization of the complement of the support of a distribution if we add some extra conditions to the pointwise convergence to zero of the symmetric means. Naturally, the uniform convergence to zero of the means on compacts of an open set $\Omega$ gives that $\Omega \subset \mathbb{R}^{n} \backslash \operatorname{supp} f$, because of the Proposition 1 ; this is the result of González Vieli and Graham [7] when $(\mathrm{T})=(\mathrm{C}, N)_{\mathrm{r}}$ for $N$ large.

Let us start with the $\phi$-transform.

Theorem 2. Let $f \in \mathcal{D}^{\prime}\left(\mathbb{R}^{n}\right)$ and let $F(\mathbf{x}, t)$ be its $\phi$-transform. Assume that $\phi(\mathbf{x}) \geq 0 \forall \mathbf{x} \in \mathbb{R}^{n}$, while $\phi(\mathbf{0})>0$. Suppose that $\phi \in$ $\mathcal{D}\left(\mathbb{R}^{n}\right)$ or that (3.17), (3.18), and (3.19) are satisfied. Suppose that pointwise

$$
\lim _{t \rightarrow 0^{+}} F(\mathbf{x}, t)=0, \quad \forall \mathbf{x} \in \Omega,
$$

where $\Omega$ is an open set. Let $p \in[1, \infty]$ and suppose that for $0<t \leq t_{0}$ the function $F(\mathbf{x}, t)$ is locally bounded in $L^{p}(\Omega)$, i.e., if $K$ is compact in $\Omega$, there exists a constant $M=M(K, p)$ such that

$$
\left(\int_{K}|F(\mathbf{x}, t)|^{p} \mathrm{~d} \mathbf{x}\right)^{1 / p} \leq M,
$$

for $p<\infty$, or if $p=\infty$,

$$
\sup \{|F(\mathbf{x}, t)|: \mathbf{x} \in K\} \leq M .
$$

Then $\Omega \subset \mathbb{R}^{n} \backslash \operatorname{supp} f$. 
Proof. It is enough to do it when $p=1$, since local boundedness in $L^{q}(\Omega)$ for $q \geq 1$ implies local boundedness in $L^{1}(\Omega)$. Now, local boundedness in $L^{1}(\Omega)$ plus distributional convergence yield that $f$ is a signed Radon measure in $\Omega$ : if $\left\{t_{n}\right\}$ is any sequence of positive numbers that converges to zero then local boundedness in $L^{1}(\Omega)$ implies that there exists a subsequence $\left\{t_{n_{k}}\right\}$ such that $F\left(\mathbf{x}, t_{n_{k}}\right)$ converges $*$-weakly in the dual space of $C(\Omega)$, that is $F\left(\mathbf{x}, t_{n_{k}}\right) \rightarrow \nu(\mathbf{x})$ where $\nu$ is a signed Radon measure in $\Omega$; but clearly $f=\nu$ in $\Omega$.

We can then write, in $\Omega, f=f_{\text {ac }}+f_{\text {dis }}+f_{\text {sin }}$, where $f_{\text {ac }}$, the absolutely continuous part, is a locally integrable function in $\Omega, f_{\text {dis }}(\mathbf{x})=$ $\sum_{\mathbf{a} \in A} c_{\mathbf{a}} \delta(\mathbf{x}-\mathbf{a})$ where $A$ is countable at the most and $\sum_{\mathbf{a} \in A \cap K}\left|c_{\mathbf{a}}\right|$ converges for all $K$ compact with $K \subset \Omega$, and where $f_{\text {sin }}$ is a continuous signed measure concentrated on a set of Lebesgue measure zero. But the distributional point value $f_{\text {ac }}(\mathbf{x})$ exists almost everywhere because $f_{\text {ac }}$ is locally integrable and equals the distributional point value $f(\mathbf{x})$ almost everywhere since $f_{\text {dis }}(\mathbf{x})=f_{\text {sin }}(\mathbf{x})=0$ almost everywhere, and from (6.1) those values are 0 , so that the function $f_{\mathrm{ac}}$ is null a.e. in $\Omega$, and so the distribution $f_{\mathrm{ac}}=0$ in $\Omega$. On the other hand, if $c_{\mathbf{a}_{0}} \neq 0$ then the contributions form $\sum_{\mathbf{a} \in A \backslash\left\{\mathbf{a}_{0}\right\}} c_{\mathbf{a}} \delta(\mathbf{x}-\mathbf{a})$ and from $f_{\text {sin }}(\mathbf{x})$ give parts of $F\left(\mathbf{a}_{0}, t\right)$ that are of order $o\left(t^{-n}\right)$ as $t \rightarrow 0^{+}$, so that the main contribution comes from $c_{\mathbf{a}_{0}} \delta\left(\mathbf{x}-\mathbf{a}_{0}\right)$, which yields $F\left(\mathbf{a}_{0}, t\right) \sim c_{\mathbf{a}_{0}} t^{-n} \phi(\mathbf{0})$ as $t \rightarrow 0^{+}$. However, this is not possible because of (6.1); hence the discrete part $f_{\text {dis }}$ also vanishes. Thus $f=f_{\text {sin }}=\mathrm{d} \mu$, a singular signed measure. We can write $\mu=\mu_{+}-\mu_{-}$, where $\mu_{ \pm}$ are positive measures, concentrated on disjoint sets, $Z_{ \pm}$. But using the results of [14, Chapter 4], the set of points $\mathbf{x}_{0}$ with infinite upper symmetric derivative

$$
\limsup _{\varepsilon \rightarrow 0^{+}} \varepsilon^{-n} \int_{\left|\mathbf{x}-\mathbf{x}_{0}\right|<\varepsilon} \mathrm{d} \mu_{ \pm}(\mathbf{x})=\infty,
$$

is of full measure with respect to $|\mu|$, and at those points, because $\phi(\mathbf{x}) \geq 0 \forall \mathbf{x} \in \mathbb{R}^{n}$ and $\phi(\mathbf{0})>0$

$$
\limsup _{\varepsilon \rightarrow 0^{+}}\left|F\left(\mathbf{x}_{0}, \varepsilon\right)\right| \geq \limsup _{\varepsilon \rightarrow 0^{+}} \varepsilon^{-n} \int_{\left|\mathbf{x}-\mathbf{x}_{0}\right|<\varepsilon} \phi(\mathbf{0}) \mathrm{d} \mu_{ \pm}(\mathbf{x})
$$

contradicting $(6.1)$; therefore $f_{\sin }=0$.

We immediately obtain a corresponding result for the characterization of the complement of the support in the Fourier inversion formula. 
Corollary 2. Let $f \in \mathcal{S}^{\prime}\left(\mathbb{R}^{n}\right)$. Suppose that pointwise

$$
\frac{1}{(2 \pi)^{n}}\left\langle\widehat{f}(\mathbf{u}), e^{-i \mathbf{u} \bullet \mathbf{x}}\right\rangle=0 \quad(\mathrm{~T}),
$$

for all $\mathbf{x} \in \Omega$, where $\Omega$ is an open set, and where $(\mathrm{T})=(\psi),(A)$, or $(C, N)_{r}$ for $N$ large. If the means are locally bounded in $L^{p}(\Omega)$ for some $p \in[1, \infty]$ then $\Omega \subset \mathbb{R}^{n} \backslash \operatorname{supp} f$.

\section{REFERENCES}

[1] Drozhzhinov, Y.N. and Zavyalov, B.I., Tauberian theorems for generalized functions with values in Banach spaces, Izv. Ross. Acad. Nauk Ser. Mat. 66 (2002), 47-118 (in Russian); translation in: Izv. Math. 66 (2002), 701-769.

[2] Estrada, R., Characterization of the Fourier series of distributions having a value at a point, Proc. Amer. Math. Soc. 124 (1996), 1205-1212.

[3] Estrada, R., The Cesàro behaviour of distributions, Proc. Roy. Soc. London Ser. A 454 (1998), 2425-2443.

[4] Estrada, R., A distributional version of the Ferenc Lukács theorem, Sarajevo J. Math. 1 (2005), 75-92.

[5] Estrada, R. and Kanwal, R.P., A distributional approach to Asymptotics. Theory and Applications, second edition, Birkhäuser, Boston, 2002.

[6] González Vieli, F.J., Inversion de Fourier ponctuelle des distributions à support compact, Arch. Math. 75 (2000), 290-298.

[7] González Vieli, F.J. and Graham, C.C., On the support of tempered distributions, Arch. Math. 88 (2007), 133-142.

[8] Graham, C.C., The support of pseudomeasures in $\mathbb{R}$, Math. Proc. Camb. Phil. Soc. 142 (2007), 149-152.

[9] Graham, C.C., The support of tempered distributions, Math. Proc. Camb. Phil. Soc. 144 (2008), 495-498.

[10] Kahane, J.P. and Salem, R., Ensembles Parfaits et Séries Trigonométriques, Hermann, Paris, 1963.

[11] Eojasiewicz, S., Sur la valeur et la limite d'une distribution en un point, Studia Math. 16 (1957), 1-36.

[12] Łojasiewicz, S., Sur la fixation des variables dans une distribution, Studia Math. 17 (1958), 1-64.

[13] Pilipović, S., On the behavior of a distribution at the origin, Math. Nachr. 141 (1989), 27-32.

[14] Saks, S., Theory of the Integral, $2^{\text {nd }}$ edition, Dover, New York, 1964.

[15] Vindas, J. and Estrada, R., Distributionally regulated functions, Studia Math. 181 (2007), 211-236.

[16] Vindas, J. and Estrada, R., Distributional point values and convergence of Fourier series and integrals, J. Fourier Anal. Appls. 13 (2007), 551-576.

[17] Vindas, J. and Pilipović, S., Structural theorems for quasiasymptotics of distributions at the origin, to appear in Math. Nachr.

[18] Walter, G., Pointwise convergence of distribution expansions, Studia Math. 26 (1966), 143-154. 
Department of Mathematics, Louisana State University, Baton Rouge, LA 70803, USA.

E-mail address: jvindas@math.lsu.edu, restrada@math.lsu.edu 